\title{
Peak Area Consistency Evaluation in Gamma Spectrometry
}

\author{
Henrik Persson ${ }^{1}$ and Kara Phillips ${ }^{1}$ \\ ${ }^{1}$ Mirion Technologies (Canberra) Inc., USA \\ hpersson@mirion.com
}

\begin{abstract}
Quantification of radionuclide activities in gamma spectrometry can be a challenging task. It depends on efficiency calibration, peak area calculation, nuclide decay data and correction factors, such as attenuation correction or true coincidence summing corrections. These quantities can present significant challenges to an accurate analysis. It is therefore desirable to have a way of assessing the quality of the radionuclide quantification that can be applied to samples with unknown activities and radionuclide compositions. A verification of the self-consistency of the analysis is one possible way of accomplishing this. In gamma spectrometry it is possible to calculate radionuclide activities using information from multiple gamma emission energies. This leads to an overdetermined system for which the solution can be used to look for inconsistencies. By calculating the recovered peak areas from the radionuclide activities and comparing these to the measured peak areas, outliers can be identified and by resolving these inconsistencies the analysis of the spectrum can be improved. This peak area consistency evaluation can be used to find incorrect shape of the efficiency calibration, missing interferences in the nuclide decay data, and point to peaks where the peak area calculation needs to be optimized. The performance of the method has been shown on a simple spectrum consisting of three radionuclides that are interfering with each other as well as a complex spectrum with unknown radionuclide composition and activities. The method will be integrated into a future version the Genie 2000 Gamma Spectroscopy Software.
\end{abstract}

Keywords -Gamma Spectrometry, Self-consistency check, Genie 2000.

\section{INTRODUCTION}

$\mathrm{G}$ AMMA spectrometry is a non-destructive measurement method for identifying and quantifying the activities of gamma-ray emitting radionuclides in a wide variety of samples including radioactive waste, environmental, and radio pharmaceutical.

A typical gamma spectrometry analysis contains several components: peak location, peak area calculation, background subtraction, nuclide identification and activity evaluation, as well as detection limits determination [1]. The main inputs to the nuclide identification and activity calculation are the peak energies and background corrected peak areas, nuclide decay data, and efficiency calibration. Each of these present a significant challenge to the gamma spectrometrist and can be the cause of inconsistencies in the analysis. Some of the considerations that need treatment are, peak area calculations need to accurately account for the shape of continuum under the peak. The detection efficiency of the sample can be different than the calibration standard or mathematical model used for the efficiency calibration. Finally, care needs to be taken when choosing the radionuclides and their emission energies to include in the nuclide library used in the analysis.

Many radionuclides emit gamma rays with different energies which creates peaks at different locations in the spectrum. The activity can be calculated from each of these peaks, and it should be the same within the uncertainties of the method. The line activities calculated from the peaks under the assumption that all counts in the peak originates from the radionuclide in question has previously been used for consistency test. However, this assumption is not fulfilled when two or more radionuclide emits energies that cannot be resolved by the detector. The radionuclide activity is in this case calculated from a least-squares fit of the activities that reproduces the measured peak areas. The peak area consistency evaluation (PACE) method relies on how well the measured peak areas are reproduced from the contribution from all radionuclides contributing to the peak. The recovered peak area is then compared to the measured peak area for each peak, and they need to be within the uncertainties for the analysis to be selfconsistent. Self-consistency is a necessary but not sufficient condition for a correct analysis of the gamma spectrum. This method is applicable for complex spectra where more than one peak contributes to the peaks in the gamma spectrum.

The PACE method only uses information from input calibrations and the measured spectrum, and therefore it can be used on measured spectra with unknown activities and radionuclide compositions. The method will be included in the Genie 2000 Gamma Spectroscopy Software.

\section{METHOD}

The weighted mean activity, A, of radionuclide emitting $n$ gamma-rays with different energies and that do not have any interferences are calculated from 


$$
A=\frac{\sum_{i=1}^{n} \frac{L_{i}}{\sigma_{i}^{2}}}{\sum_{i=1}^{n} \frac{1}{\sigma_{i}^{2}}}
$$

in Genie 2000 [2] where $L_{i}$ is the line activity of line at peak $\mathrm{i}$ with an uncertainty of $\sigma_{i}$. The recovered peak area for the $\mathrm{i}^{\text {th }}$ peak, $P_{i}$, is then calculated from

$$
P_{i}=A \varepsilon_{i} I_{i} T
$$

where $\varepsilon_{i}$ is the efficiency at the energy of the peak, $I_{i}$ is the intensity of the line contributing to the peak and $\mathrm{T}$ is the live time of the acquisition. The uncertainty in the recovered peak area is

$$
\sigma_{P_{i}}=\mathrm{P} \sqrt{\left(\frac{\sigma_{A_{i}}}{A}\right)^{2}+\left(\frac{\sigma_{\varepsilon_{i}}}{\varepsilon_{i}}\right)^{2}+\left(\frac{\left.\sigma_{I_{i}}\right)}{I_{i}}\right)^{2}}
$$

For radionuclides that have at least one of its line interfering with one or more other radionuclides the activity is obtained from minimizing the $\chi^{2}$ function

$$
\chi^{2}=\sum_{i=1}^{n} \frac{\left(y_{i}-\sum_{j=1}^{m} I_{i j} x_{j}\right)^{2}}{\sigma_{i}^{2}},
$$

where $\mathrm{n}$ is the number of peaks in the interference, $\mathrm{m}$ is the number of radionuclides in the interference, $y_{i}=\frac{N_{i}}{\varepsilon_{i} T}, N_{i}$ is the measured peak area for peak $\mathrm{i}, I_{i j}$ is the intensity of radionuclide $\mathrm{j}$ for peak $\mathrm{i}$. The recovered peak areas are calculated from the minimum of (4).

With an uncertainty of

$$
P_{i}=\varepsilon_{i} T \sum_{j=1}^{m} I_{i j} x_{j}
$$

$\sigma_{P_{i}}=\sqrt{\left(T \sum_{j=1}^{m} I_{i j} x_{j}\right)^{2} \sigma_{\varepsilon_{i}}^{2}+\varepsilon_{i}^{2} T^{2} \sum_{j=1}^{m}\left(I_{i j}^{2} \sigma_{x_{j}}^{2}+x_{j}^{2} \sigma_{I_{i j}}^{2}\right)}$

Finally, a z-score can be defined as the difference between the recovered and measured peak area divided by their combined uncertainty

$$
z_{i}=\frac{P_{i}-N_{i}}{\sqrt{\sigma_{P_{i}}^{2}+\sigma_{N_{i}}^{2}}}
$$

which makes it possible to define an outlier peak if the absolute value of the $\mathrm{z}$-score is larger than 3 .

This makes it possible to find the peaks for which the analysis is not accounting for the counts for a peak while minimizing the number of falsely labeled peaks that are not outliers.

The outliers that are found using this method should be investigated for possible causes of the inconsistency and the reason for the inconsistency should be resolved. Failing that a conscious decision can be made to exclude the peak from the analysis if the reason cannot be resolved so that the activities of the identified radionuclides are not affected by the outlier.

\section{ANALYSIS}

The PACE method has been applied to two example gamma spectra, the first is a known spectrum containing three radionuclides that all forms an interference and no other radionuclides. The purpose of this spectrum is to give examples of signatures that show up in the PACE analysis. The second spectrum is a complex spectrum with unknown activities and radionuclide composition and shows how PACE can be used to improve the analysis of unknown spectra.

\section{A. Known spectrum}

The first example that the PACE method was applied to is a spectrum containing Co-57, Se-75, and Eu-152. The spectrum was generated by MCNP-CP [3]. The advantage of using a simulated spectrum is that the spectrum is fully known and there are no unknown radionuclides contributing to the spectrum. All three radionuclides contribute to the $121 \mathrm{keV}$ peak and therefore consist of an interference set. Table 1 shows the energy of the peaks included in the analysis and which radionuclide contribute to each peak. Both peaks of Co-57 have contribution from at least one of the other two radionuclides, and the activity is inferred by excess counts in the 121 and 136 keV peaks.

TABLE 1

THE PEAKS USED FOR ACTIVITY DETERMINATION AND THE ASSOCIATED RADIONUCLIDES FOR THE CO-57, SE-75, AND EU-152 SPECTRUM

\begin{tabular}{cccc}
\hline $\begin{array}{c}\text { Peak Energy } \\
(\mathrm{keV})\end{array}$ & $\mathrm{Co}-57$ & $\mathrm{Se}-75$ & $\mathrm{Eu}-152$ \\
\hline 40 & & $\mathrm{X}$ & $\mathrm{X}$ \\
66 & & $\mathrm{X}$ & \\
99 & $\mathrm{X}$ & $\mathrm{X}$ & $\mathrm{X}$ \\
121 & $\mathrm{X}$ & $\mathrm{X}$ & \\
136 & & $\mathrm{X}$ & $\mathrm{X}$ \\
244 & & $\mathrm{X}$ & \\
264 & & & \\
279 & & $\mathrm{X}$ & $\mathrm{X}$ \\
344 & & $\mathrm{X}$ \\
400 & & & $\mathrm{X}$ \\
778 & & $\mathrm{X}$ \\
964 & & \\
1085 & & & \\
1408 & & & \\
\hline \hline
\end{tabular}

The peak area recovery z-score was calculated using (7) for the peaks in Table 1 and is shown as a function of energy in Figure 1a. The absolute peak area recovery z-score is less than 2 for all peaks and there is no trend in the ratio as a function of energy, indicating that the analysis is self-consistent, as expected for a known spectrum. Figure 1b shows the line activity ratio, defined as the line activity divided by the weighted mean activity for the three radionuclides. The line activity ratio is not within the uncertainties for the 121 and 136 $\mathrm{keV}$ peaks. These are the peaks for which multiple radionuclides contribute to. This demonstrates that the PACE method is a better measure of self-consistency for radionuclides that interfere with each other. 

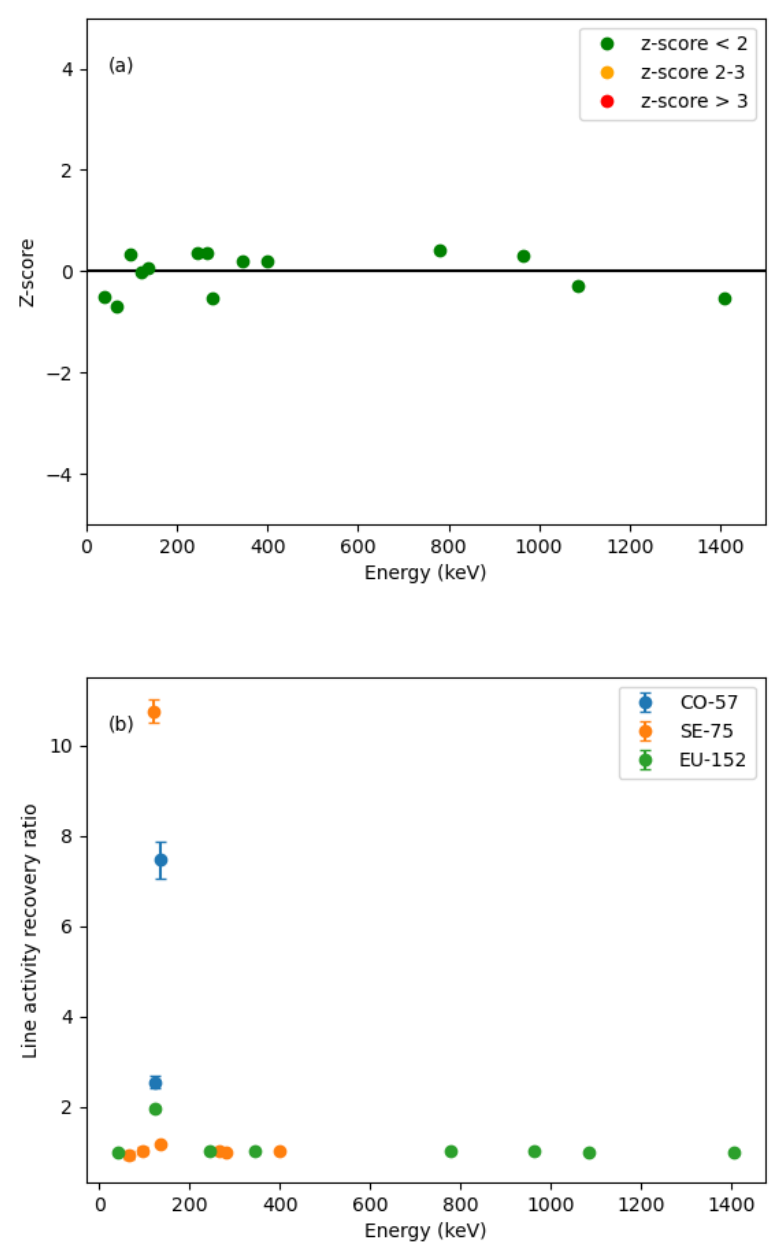

Figure 1, The Peak Area recovery z-score (a) and Line activity ratio (b) for the interference consisting of Co-57, Se-75, and Eu-152.

Different causes for inconsistencies can create different signatures in the peak area recovery ratio. One possible cause of inconsistency is that the shape of the efficiency calibration is different than the shape of the sample efficiency. To demonstrate this signature, the efficiency calibration used for the analysis of the Co-57, Se-75, and Eu-152 was increased for lower energies emulating analyzing a sample with increased attenuation compared to the efficiency calibration. Figure 2 shows the z-score for the analysis with the altered efficiency calibration. The z-score for low energies decreases for when the efficiency calibration is higher than the sample efficiency, which is seen in the decrease in the ratio for the 40, 66, and 99 $\mathrm{keV}$ peaks compared to the ratios in Figure 1a. The uncertainties for the 66 and $99 \mathrm{keV}$ are large, which brings up the $\mathrm{z}$-score to a value between negative 2 and 3 while the $\mathrm{z}$ score for the $39 \mathrm{keV}$ is clearly below negative 3 . This shows that the method works better when the uncertainties are small for most of the peaks. The least squares solution decreases the activity of Co-57, hiding the effect for these two peaks, demonstrating that radionuclides that do not have any peaks that are free from interferences from other radionuclides can hide the signature in the $\mathrm{z}$-score. To a smaller degree the $\mathrm{z}$-score increases at higher energies because the peaks at low energy decreases the radionuclide activity which increases the recovery ratio. The solution to this signature is to adjust the modeled geometry if the efficiency calibration is done mathematically. For measured efficiency calibrations, an attenuation correction is required.

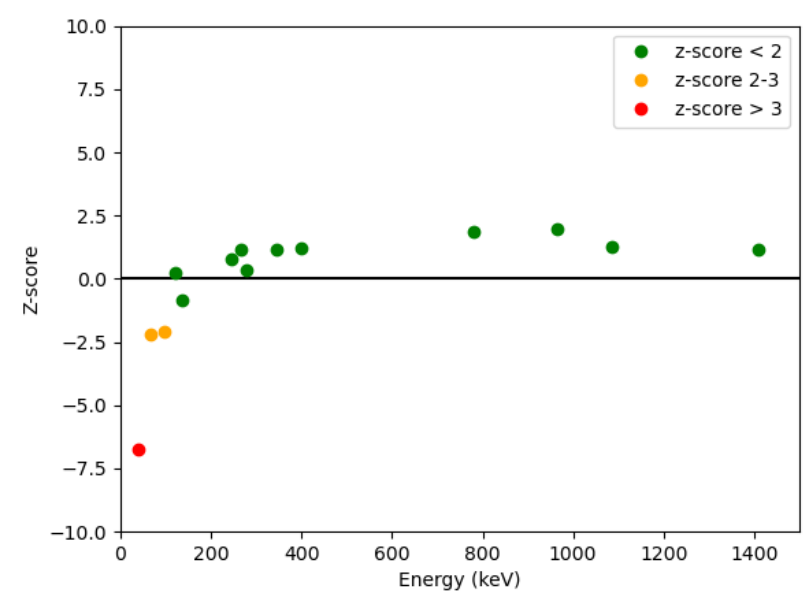

Figure 2, The peak area z-score for an analysis where the shape of the efficiency calibration is different from the shape of the sample efficiency

Another common cause for inconsistency is missing interferences in the nuclide library. Missing interferences can be missing lines from identified radionuclides, such as low intensity lines omitted from high activity radionuclides Alternatively, they could be radionuclides missing from the nuclide library. This is demonstrated in Figure 3 where the same spectrum has been analyzed with Co-57 removed from the nuclide library. The signature of missing interferences is a $\mathrm{z}$ score larger than 3 for the peaks that are missing an interference and there is no correlation to peaks close in energy, as was the case for the shape of the efficiency calibration. The size of the increase in the z-score depends on the activity and intensity of the missing radionuclide, the contribution from the identified radionuclides, and the uncertainty of the peak area. The increase is largest for high intensity lines where the missing radionuclides have a large contribution. For Co-57 the increase is highest for the $121 \mathrm{keV}$ peak because of the much higher intensity compared to the $136 \mathrm{keV}$ line. Another consequence of missing interferences is that the other radionuclides contributing to the peak will have an overestimated activity and therefore the $\mathrm{z}$-score will be lower than 1 as can be seen in Figure 3 for the peaks without contribution from Co-57 causing the $279 \mathrm{keV}$ and 1408 peaks to have a $\mathrm{z}$-score below negative 2. There are several other possible causes of inconsistency in gamma spectrometry analysis and some of them are listed with a suggested solution in Table 1 . 


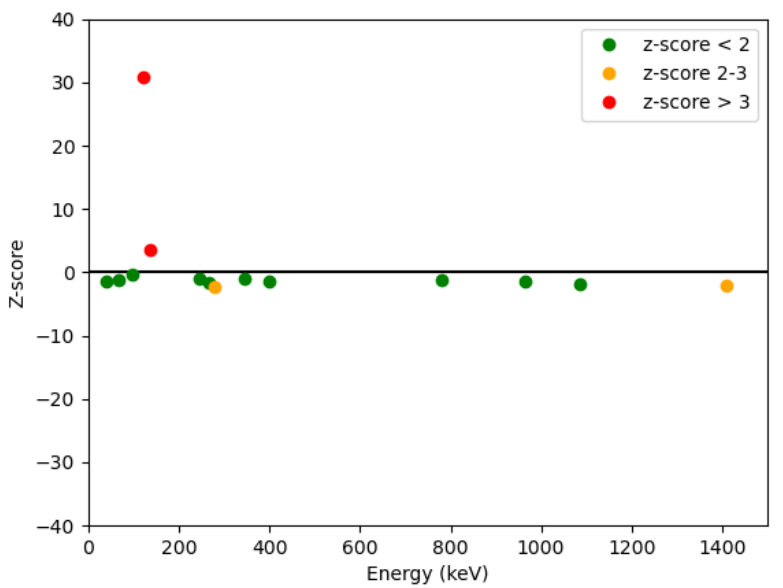

Figure 3, The peak area recovery z-score for an analysis of an interference where a contributing interference is missing.

TABLE 2 COMMON CAUSES FOR INCONSISTENCIES AND THEIR SIGNATURES IN THE PEAK AREA RECOVERY RATIO.

\begin{tabular}{|c|c|c|}
\hline Cause & Signature & Suggested solution \\
\hline $\begin{array}{c}\text { Efficiency shape } \\
\text { high/low at low energies }\end{array}$ & $\begin{array}{c}\text { Decrease } \\
\text { /increase at low } \\
\text { energy }\end{array}$ & $\begin{array}{c}\text { Refine model or } \\
\text { perform attenuation } \\
\text { correction }\end{array}$ \\
\hline Missing interference & $\begin{array}{l}\text { Increase at the } \\
\text { peak missing the } \\
\text { interference }\end{array}$ & $\begin{array}{l}\text { Add line or nuclide } \\
\text { to library }\end{array}$ \\
\hline $\begin{array}{c}\text { Missing true } \\
\text { coincidence summing } \\
\text { correction }\end{array}$ & $\begin{array}{c}\text { Decrease } \\
\text { /increase in ratio } \\
\text { when summing } \\
\text { in/out is } \\
\text { dominating }\end{array}$ & $\begin{array}{l}\text { Add correction } \\
\text { factor or don't } \\
\text { include in analysis }\end{array}$ \\
\hline Peak fitting & $\begin{array}{l}\text { Increase or } \\
\text { decrease of } \\
\text { recovery ratio }\end{array}$ & $\begin{array}{l}\text { Improve peak } \\
\text { fitting }\end{array}$ \\
\hline $\begin{array}{l}\text { Single/double escape } \\
\text { interference }\end{array}$ & $\begin{array}{l}\text { Increase at the } \\
\text { escape } \\
\text { interference }\end{array}$ & $\begin{array}{l}\text { Calculate escape } \\
\text { efficiency or } \\
\text { exclude from } \\
\text { analysis }\end{array}$ \\
\hline $\begin{array}{c}\text { Magnitude of efficiency } \\
\text { calibration }\end{array}$ & No signature & N/A \\
\hline
\end{tabular}

\section{B. Unknown spectrum}

To demonstrate how the PACE method can be used to improve the analysis of an unknown spectrum, it was applied to the analysis of a complex spectrum of primary reactor coolant measured by a HPGe detector shortly after leaving the reactor. The radionuclide composition and their activities were unknown at the time of analysis. The measured spectrum is shown in Figure 4.

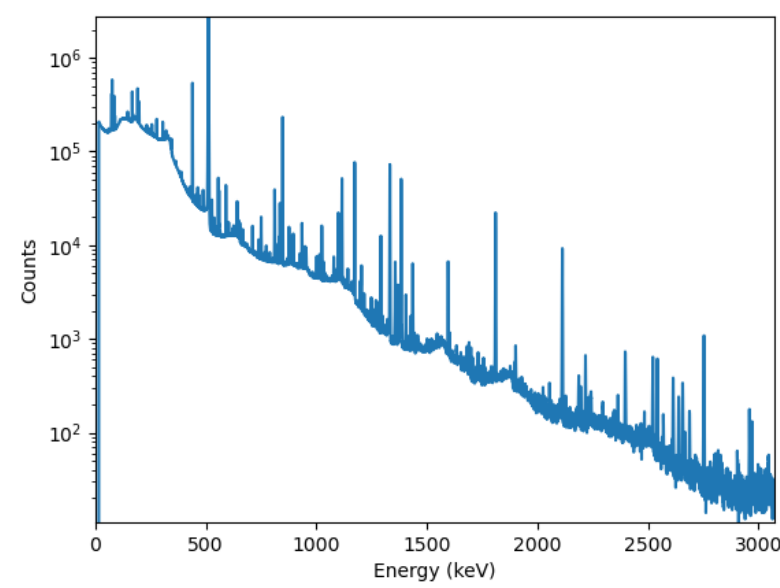

Figure 4, the reactor coolant spectrum.

There were 278 peaks found in the spectrum, with a large interference containing 139 peaks and 30 radionuclides. The peak area recovery ratios for the interference are shown in Figure 5. There are 22 outlier peaks where the absolute value of the $\mathrm{z}$-score is larger than 3,19 of these have a z-score larger than 3 and 3 had a ratio below negative 3 . All the peaks that were identified as outliers were investigated for common reasons according to Table 2 .

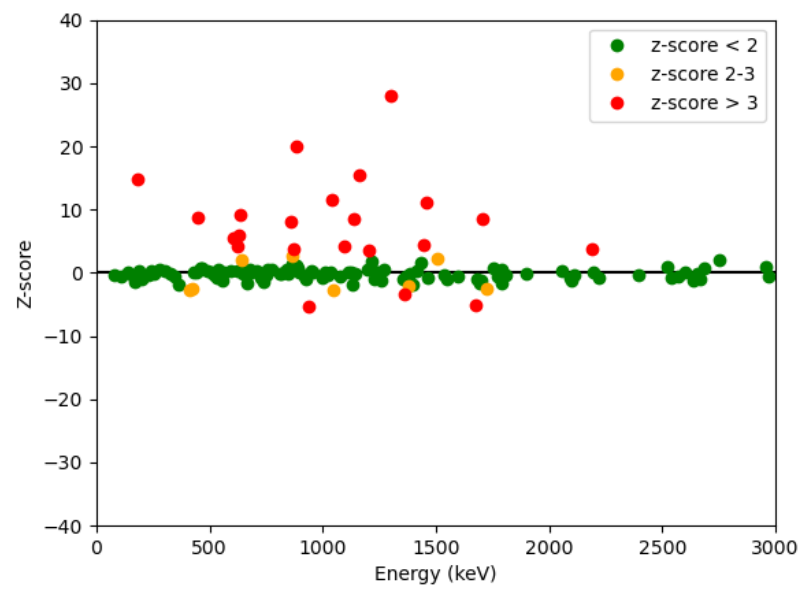

Figure 5. The peak area recovery z-score for the complex spectrum.

There is no noticeable energy dependence in the peak area recovery ratio indicating that the shape of the efficiency calibration, performed using ISOCS efficiency modeling software [4], is self-consistent. The efficiency is low enough that true coincidence summing can be neglected. The peaks were checked for peak fitting, missing interferences, and single and double escape interferences. The cause for the outliers is listed in Table 3. For one of the peaks, peak fitting was determined to be the cause of the inconsistency. It was determined that 12 peaks had missing interferences from radionuclides that were already identified in the nuclide library. Six of these were from low intensity lines from Sr-93. More importantly, the cause of 3 of the outliers were determined to be interference from radionuclides that were missing from the 
nuclide library and these radionuclides would not have been identified if the self-consistency analysis had not been performed. Additionally, the cause for one of the peaks were interference from single escape from a higher energy peak. For the peaks with a z-score lower than negative 3 the cause was determined to be overestimated activity of $\mathrm{Ag}-110 \mathrm{~m}$ because of missing interferences for the 446, 620, and $884 \mathrm{keV}$ peaks and overestimation of La-142 because of missing interference for the $1160 \mathrm{keV}$ peak, and overestimation of I-135 caused by missing interference for the 1040,1458 , and $1706 \mathrm{keV}$ peaks.

\begin{tabular}{cc}
\begin{tabular}{c} 
TABLE 3 THE OUTLIER PEAKS AND THE CAUSE DETERMINED FOR THE OUTLIER \\
\hline \hline $\begin{array}{c}\text { Peak energy } \\
(\mathrm{keV})\end{array}$
\end{tabular} \\
\hline 184 & Cause \\
446 & Peak fitting \\
602 & Missing line from Sr-93, I-132 \\
620 & Cs-140 missing from library \\
630 & Missing line from I-132 \\
635 & Missing line from Sr-91, Sr-93 \\
857 & Missing line from Ba-141 \\
871 & Missing line from Sr-91 \\
884 & Rb-90m missing from library \\
937 & I-134 missing from library \\
1040 & Overestimation of Ag-110m activity \\
1093 & Missing line from Sr-93 \\
1136 & Missing line from Sr-93 and Ba-142 \\
1160 & Missing line from Sr-93 and I-134 missing from \\
1203 & library \\
1299 & Missing line from I-135 and Ba-141 \\
1362 & Missing line from Y-91 and Cs-138 \\
1444 & Interference from single escape peak \\
1458 & Overestimation of La-142 activity \\
1677 & Missing lines from Sb-124, I-132. Cs-138 and \\
1706 & La-142 \\
2187 & Missing line from I-135 \\
\hline & Overestimation of I-135 \\
& Missing line from Sr-93 \\
\hline & Unknown \\
\hline
\end{tabular}

\section{CONCLUSIONS}

PACE is a method for analyzing self-consistency in gamma spectrometry that has been developed for inclusion in the Genie-2000 Gamma Spectroscopy Software. The method relies on the fact that it is possible to calculate radionuclide activities from multiple inputs from a single spectrum. The radionuclide activities are then used to calculate the peak areas recovered from the radionuclide activities and compared to the measured peak areas. The method has been demonstrated on a known sample and signatures as well as suggested solutions for several common causes for inconsistencies were discussed. The method uses no other information than what is available from the calibrations and the spectrum and can therefore be applied to spectra with unknown radionuclide activity and composition. This was demonstrated for an analysis of an unknown sample of primary coolant measured shortly after being removed from the reactor. The analysis revealed that 3 radionuclides were missing from nuclide library and that several radionuclides were missing lines contributing to the peaks in the spectrum. It also revealed that the activity was overestimated for three radionuclides.

\section{REFERENCES}

[1] J Verplancke, et al, "Semiconductor Detectors," in Handbook of Radioactivity Analysis, M. F. L'Annunziata, $3^{\text {rd }}$ ed, Amsterdam, The Netherlands: Elsevier, 2012, pp. 342-360.

[2] Genie 2000 Spectroscopy Software Customization Tools, Mirion Technologies (Canberra) Inc., Meriden, CT, USA, 2013.

[3] A.N. Berlizov, "MCNP-CP: a correlated particle radiation source extension of a general purpose Monte Carlo N-Particle transport code" ACS Symposium series., vol. 945, pp. 183-194, Nov. 2006. https://pubs.acs.org/doi/abs/10.1021/bk-2007-0945.ch013

[4] R Venkataraman, F Bronson, V Abashkevich, B.M Young, M Field, "Validation of in situ object counting system (ISOCS) mathematical efficiency calibration software", NIMA, vol. 422, pp 450-454, Feb 1999, https://doi.org/10.1016/S0168-9002(98)01115-2 